This is a self-archived version of an original article. This version may differ from the original in pagination and typographic details.

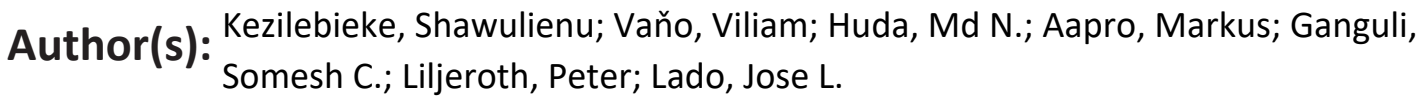

Title: Moiré-Enabled Topological Superconductivity

Year: 2022

Version: Published version

Copyright: (c) 2022 the Authors

Rights: $C C B Y 4.0$

Rights url: https://creativecommons.org/licenses/by/4.0/

Please cite the original version:

Kezilebieke, S., Vaňo, V., Huda, M. N., Aapro, M., Ganguli, S. C., Liljeroth, P., \& Lado, J. L. (2022).

Moiré-Enabled Topological Superconductivity. Nano Letters, 22(1), 328-333.

https://doi.org/10.1021/acs.nanolett.1c03856 


\title{
Moiré-Enabled Topological Superconductivity
}

\author{
Shawulienu Kezilebieke,* Viliam Vaňo, Md N. Huda, Markus Aapro, Somesh C. Ganguli, Peter Liljeroth,
} and Jose L. Lado*

Cite This: https://doi.org/10.1021/acs.nanolett.1c03856

Read Online

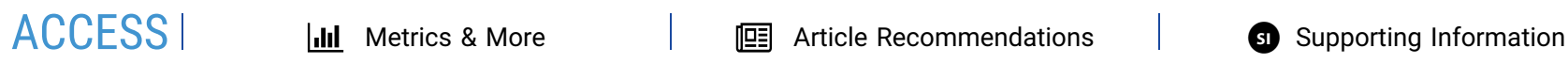
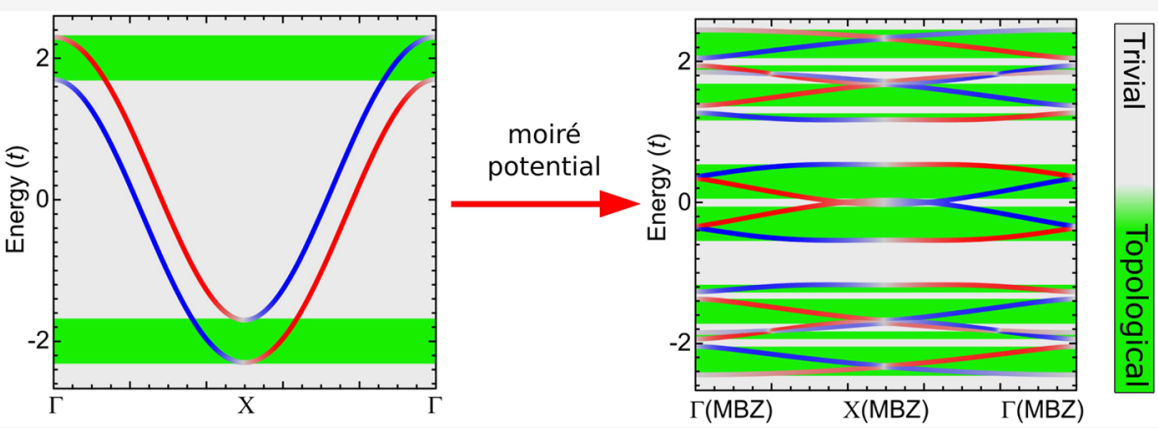

ABSTRACT: The search for artificial topological superconductivity has been limited by the stringent conditions required for its emergence. As exemplified by the recent discoveries of various correlated electronic states in twisted van der Waals materials, moiré patterns can act as a powerful knob to create artificial electronic structures. Here, we demonstrate that a moiré pattern between a van der Waals superconductor and a monolayer ferromagnet creates a periodic potential modulation that enables the realization of a topological superconducting state that would not be accessible in the absence of the moiré. The magnetic moiré pattern gives rise to $\mathrm{Yu}$-Shiba-Rusinov minibands and periodic modulation of the Majorana edge modes that we detect using low-temperature scanning tunneling microscopy (STM) and spectroscopy (STS). Moiré patterns and, more broadly, periodic potential modulations are powerful tools to overcome the conventional constraints for realizing and controlling topological superconductivity.

KEYWORDS: scanning tunneling microscopy, topological superconductor, moiré pattern, 2D ferromagnet

$\mathrm{T}^{\mathrm{h}}$ here are many routes to realizing topological superconductivity in artificial structures, ${ }^{1-10}$ and perhaps the most widely used path uses the combination of superconductivity, spin-orbit coupling, and magnetism. ${ }^{11,12}$ This recipe has been recently used in a $\mathrm{CrBr}_{3} / \mathrm{NbSe}_{2}$ van der Waals $(\mathrm{vdW})$ heterostructure, where the emergence of topological superconductivity was demonstrated. ${ }^{13}$ In stark contrast with the realization based on semiconducting nanowires, ${ }^{14-16}$ the electronic structure of the $\mathrm{CrBr}_{3} / \mathrm{NbSe}_{2}$ system features a highly doped band of $\mathrm{NbSe}_{2}$, far from the typical allowed regimes for topological superconductivity to appear. This system has a complex electronic structure combining a Fermi surface reconstruction of $\mathrm{NbSe}_{2}$ stemming from its charge density wave, ${ }^{17}$ together with a strong moire arising from the lattice mismatch between $\mathrm{NbSe}_{2}$ and $\mathrm{CrBr}_{3}$. It is surprising that such complex electronic structure with no external control parameters turns out to give rise to a state featuring topological superconductivity. The emergence of topological superconductivity in a $\mathrm{NbSe}_{2} / \mathrm{CrBr}_{3}$ heterostructure ${ }^{13}$ can be rationalized as follows. The ferromagnetic state of $\mathrm{CrBr}_{3}$ induces an exchange field on the electronic structure of $\mathrm{NbSe}_{2}$, and the mirror symmetry breaking of the heterostructure creates a strong Rashba spin-orbit coupling in the
$\mathrm{NbSe}_{2}$ bands. When including the intrinsic s-wave superconducting order of $\mathrm{NbSe}_{2}$, the low energy electronic structure harvests the three fundamental ingredients for the emergence of artificial topological superconductivity: ${ }^{1-10} \mathrm{~s}$-wave superconducting order, Rashba spin-orbit coupling, and exchange fields. Such low-energy effective model has been shown to faithfully capture the phenomenology observed experimentally. ${ }^{13}$ However, interesting additional microscopic contributions have been so far unaddressed. First, Majorana edge modes showed strong regular modulation at the edges of the topological superconducting island. Second, the mismatch between the $\mathrm{NbSe}_{2}$ and $\mathrm{CrBr}_{3}$ monolayers gives rise to a moiré pattern modulating all the parameters in space. And finally, the emergence of topological superconductivity in the minimal model required a delicate fine-tuning of the $\mathrm{NbSe}_{2}$ Fermi level. Here we extend our earlier experimental results on the $\mathrm{CrBr}_{3}$ /

Received: October 6, 2021

Revised: December 22, 2021 
a
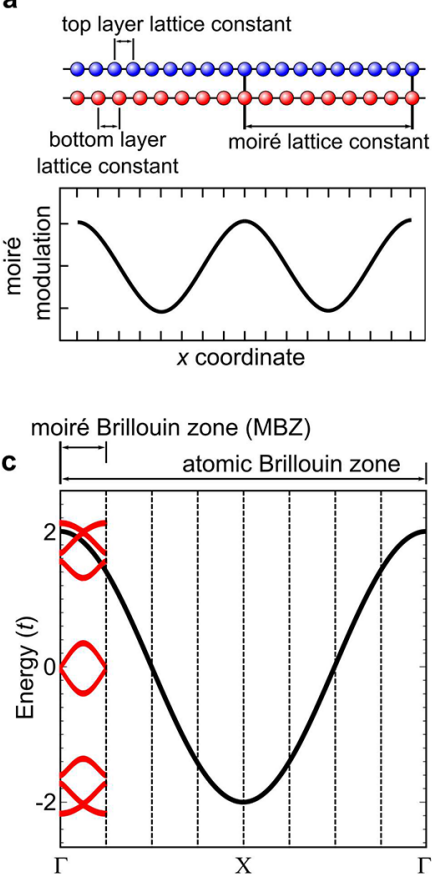

b

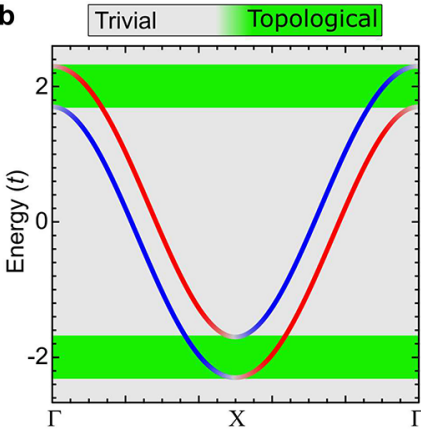

d

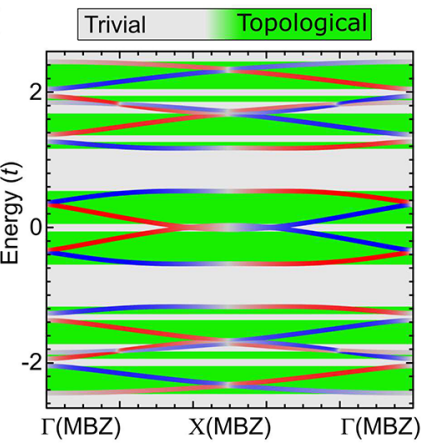

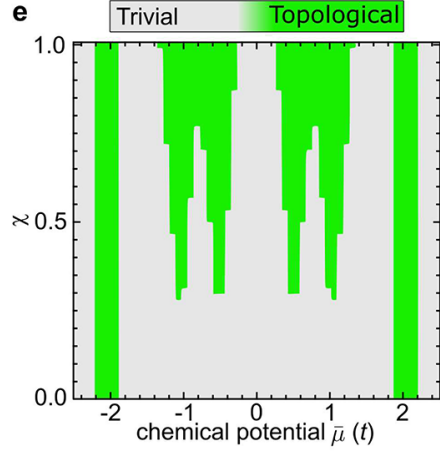

f

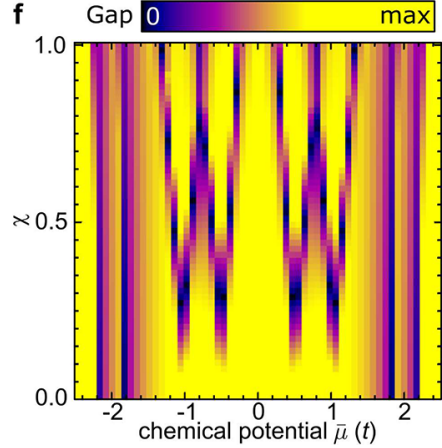

Figure 1. Moiré-driven topological superconductivity. (a) Schematic of the moire pattern set up by the lattice mismatch, and the resulting modulation of the on-site energies, the exchange coupling, and other parameters depending on the registry between the two lattices. (b) Pseudohelical states appear in the presence of Rashba and exchange interactions at the top and bottom of the band of a one-dimensional model, giving rise to topological superconductivity (green). (c) Band structure of a simple one-dimensional model before (black line) and after (red lines) turning on the moire modulation. (d) Including the effect of the moire pattern enables pseudohelical states and hence topological superconductivity to emerge at the top and bottom of the moire minibands. Here, the moire modulation is given by a modulation of the on-site energies. (e, f) Topological phase diagram (e) and the gap (f) for a 1D chain as a function of the $\bar{\mu}$ and the modulation $\chi$ of exchange.

$\mathrm{NbSe}_{2}$ system, demonstrating how the previous three features are naturally accounted by emergent moire phenomena of the heterostructure.

Here, we show that the apparent complexity created by the moire pattern in the $\mathrm{CrBr}_{3} / \mathrm{NbSe}_{2}$ system can be the ultimate driving force of its topological superconducting state. In particular, the strongly modulated electrostatic potential and exchange coupling in the moire heterostructure give rise to modulated $\mathrm{Yu}-$ Shiba-Rusinov (YSR) bands that allow for the emergence of topological superconductivity in generic regimes where it is otherwise forbidden. We explain theoretically and demonstrate experimentally that the moire modulation of the topological state emerging from the YSR bands is also visible in the spatial distribution of the one-dimensional topological Majorana modes. Our results put moiré physics forward as a powerful knob enabling topological superconductivity. Finally, conceptually similar effects can be realized by creating a periodic potential modulation (e.g., through external gating) in semiconducting devices, ${ }^{10,14,15,18-25}$ which offers new ways of controlling topological superconductivity toward the realization of topological qubits in the future.

To understand the potential of moire modulations for driving topological superconductivity, we consider a generic model incorporating long-wavelength modulations in its different parameters. $^{26}$ Specifically, we take a Hamiltonian that includes all the known ingredients for topological superconductivity: s-wave superconductivity, Rashba spinorbit coupling, and ferromagnetism..$^{1-3,12,27}$ We introduce the moire modulation through spatial variation of the parameters of the tight-binding model: on-site energies, the hoppings, the exchange coupling, Rashba spin-orbit coupling, and the s- wave superconductivity (details are given in the Supporting Information). Despite the increasing complexity of the Hamiltonian $H$ from having spatially dependent order parameters, their effects on enabling a topological superconducting state in arbitrary conditions can be easily rationalized. In order to illustrate these possibilities, we first focus on a minimal case: a one-dimensional moiré system (Figure 1a).

For a one-dimensional model with uniform order parameters, topological superconductivity can only appear at the top bottom of the band, as shown in Figure $1 \mathrm{~b}$. This is associated with a single set of pseudohelical states that develop at the top and bottom of the band in the presence of Rashba spin-orbit interaction and exchange coupling. Turning on a moiré modulation in the chemical potential $\mu(\mathbf{r}) \sim \cos (\Omega x)$, for a given wavevector $\Omega$, will cause folding of the band structure and opening of minigaps between the folded bands as illustrated in Figure 1c. ${ }^{26,28-30}$ As shown in Figure 1c, there are additional band tops and bottoms, where topological superconductivity can potentially be realized. Indeed, when Rashba spin-orbit coupling, exchange, and superconductivity are included in addition to the moire pattern, pseudohelical states appear close to charge neutrality (Figure 1d), allowing for the emergence of topological superconductivity. This leads to topological regions in the phase diagram (Figure 1e) at values of chemical potential corresponding to a topologically trivial state in the absence of the moire modulation. Associated with new topological regions, gap closing and reopening are driven by the moire modulation as shown in Figure 1f. It is important to emphasize that in the absence of the moire modulation, no topological superconducting state can be 


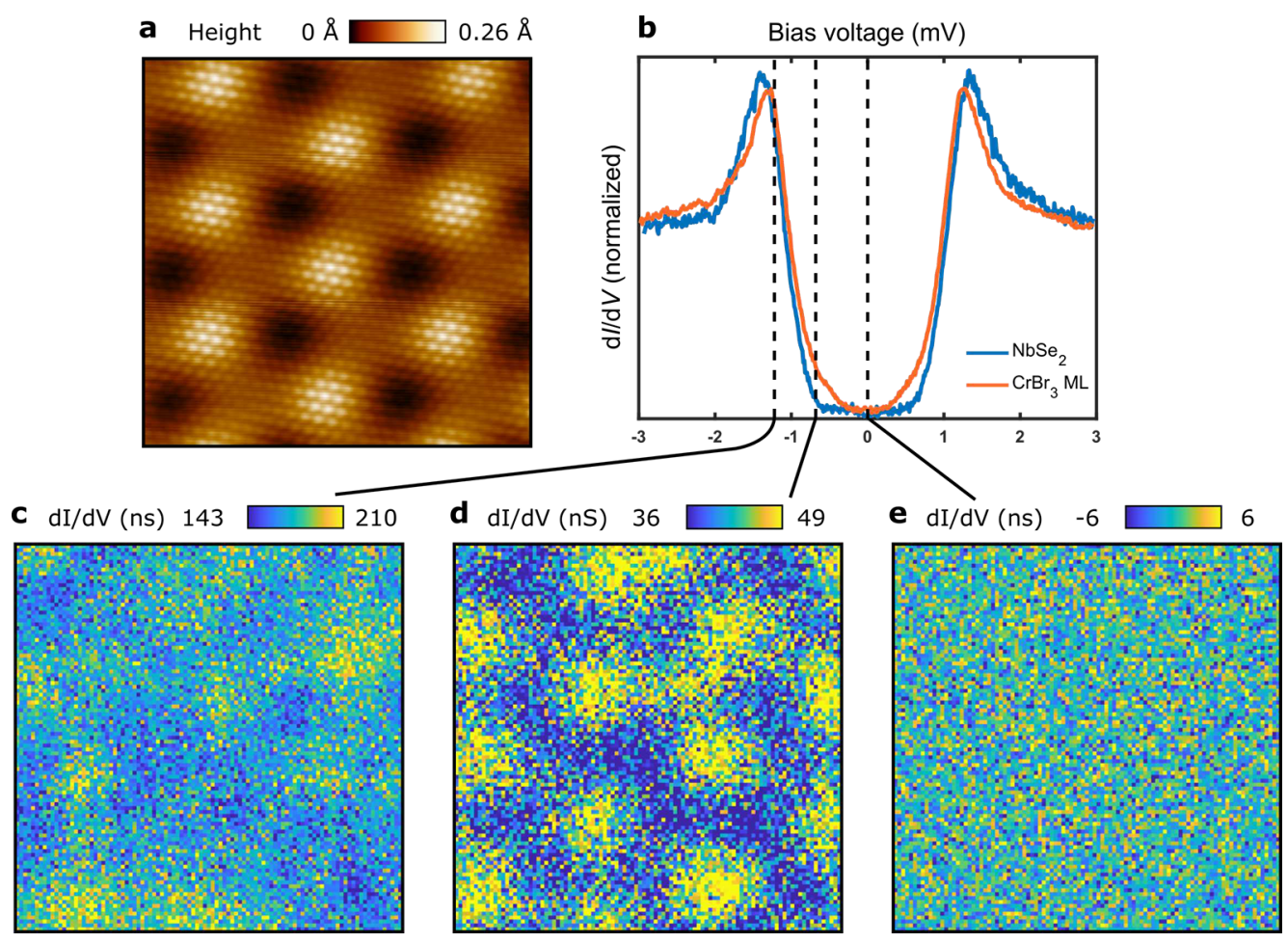

Figure 2. Correlation between moiré pattern and YSR bands. (a) STM image of $\mathrm{CrBr}_{3} \mathrm{ML}$ on $\mathrm{NbSe}_{2}$ obtained at $V=1.5 \mathrm{~V}$ and $I=300 \mathrm{pA}$. Image size is $20 \times 20 \mathrm{~nm}^{2}$. (b) $\mathrm{d} I / \mathrm{d} V$ spectroscopy on the $\mathrm{NbSe}_{2}$ substrate (blue) and on the island of $\mathrm{CrBr}_{3} \mathrm{ML}$ (orange). (c-e) $\mathrm{d} I / \mathrm{d} V$ maps (from spectral grid) at different $V$ values highlighted in (b). The maps correspond to the STM image in (a), and each map is plotted on a separate color scale. A clear correlation between the moire pattern and $\mathrm{d} I / \mathrm{d} V$ map only appears at the energy of the YSR bands $(V=0.8 \mathrm{mV})$. The grid map measurement conditions are the following: $V=3 \mathrm{mV}, I=300 \mathrm{pA}$, and $T=350 \mathrm{mK}$.

created at all in this energy range. While this example uses a modulation of the chemical potential, modulation in either exchange, Rashba, hoppings, or proximity superconductivity is effective to drive these moiré-enabled topological phase transitions (Figure S1). This idea provides a new direction to explore topological superconductivity in designed onedimensional systems, such as nanowires grown with a longrange modulation, ${ }^{31,32}$ in doping regimes in which it would not be allowed otherwise.

This phenomenology can be extended to two-dimensional systems that naturally arise due to the moiré modulation in van der Waals heterostructures. Figure $2 \mathrm{a}$ shows an atomically resolved STM image of the $\mathrm{CrBr}_{3}$ monolayer grown on a bulk $\mathrm{NbSe}_{2}$ substrate (see Supporting Information for experimental details), revealing a well-ordered moiré superstructure with 6.3 $\mathrm{nm}$ periodicity arising from the lattice mismatch between the $\mathrm{CrBr}_{3}$ and the $\mathrm{NbSe}_{2}$ layers. ${ }^{33}$ The moire pattern matches a structure with $19 \mathrm{NbSe}_{2}$ unit cells accommodating 10 unit cells of $\mathrm{CrBr}_{3}$, thus forming a $6.3 \mathrm{~nm} \times 6.3 \mathrm{~nm}$ superstructure. This also matches the measured lattice constants of $\mathrm{CrBr}_{3}$ and $\mathrm{NbSe}_{2}$.

The interaction of the magnetism of the $\mathrm{CrBr}_{3}$ layer ${ }^{13,33,34}$ with the superconductivity from the $\mathrm{NbSe}_{2}$ substrate gives rise to the YSR bands inside the superconducting gap that are also modulated by the moire pattern. The formation of YSR band is shown in Figure $2 b$ (orange line) where the $d I / d V$ spectrum taken in the middle of the $\mathrm{CrBr}_{3}$ island has a pair of conductance onsets at $\pm 0.35 \mathrm{mV}$. This spectroscopic signature can be compared to a $\mathrm{d} I / \mathrm{d} V$ spectrum of bare $\mathrm{NbSe}_{2}$, where a hard gap with an extended region of zero differential conductance around zero bias is observed (Figure 2b, blue line). By subtracting the background spectra from the twoband model fit, we obtain the experimental topological gap that is around $\Delta_{\mathrm{t}} \approx 0.3 \Delta$ (see Supporting Information). In order to visualize the spatial modulation of the YSR band, we have recorded grid $\mathrm{d} I / \mathrm{d} V$ spectroscopy maps (Figure $2 \mathrm{c}-\mathrm{e}$ ) over the area shown in the Figure 2a. The $\mathrm{d} I / \mathrm{d} V$ maps exhibit periodic modulation of the signal intensity over the moire unit cell only at the energy of the YSR bands. This is caused by the intensity variations of the YSR band local density of states (LDOS) rather than energy variations of the YSR band as further demonstrated in the Supporting Information (Figures $\mathrm{S} 7$ and S8).

The microscopic origin of the variations of the YSR band intensities can be easily rationalized. First, the modulation of exchange (Figure 3a) stems from the strong dependence of superexchange interactions on the local stacking, as demonstrated in $\mathrm{CrI}_{3}$ and $\mathrm{CrBr}_{3}$ bilayers. ${ }^{32,35,36}$ This feature suggests that the moire pattern not only modulates the absolute value of the effective exchange but also can change its sign. ${ }^{35,36}$ Second, as a consequence of the modulation of the exchange field, the superconducting order parameter will also be modulated in the opposite way, due to the competition of s-wave superconductivity from $\mathrm{NbSe}_{2}$ and the proximity induced exchange field. ${ }^{37}$ Third, the modulation of the onsite energies stems from an electrostatic effect associated with the stacking. We can directly measure the modulation of this electrostatic potential through the spatial modulation of the conduction band edge of $\mathrm{CrBr}_{3}$ (Figure S9). Fourth, modulations in the hoppings are expected from small relaxation effects, wellknown in other dichalcogenide-based twisted systems. ${ }^{38,39}$ Fifth, the charge density wave of $\mathrm{NbSe}_{2}{ }^{17}$ will introduce 

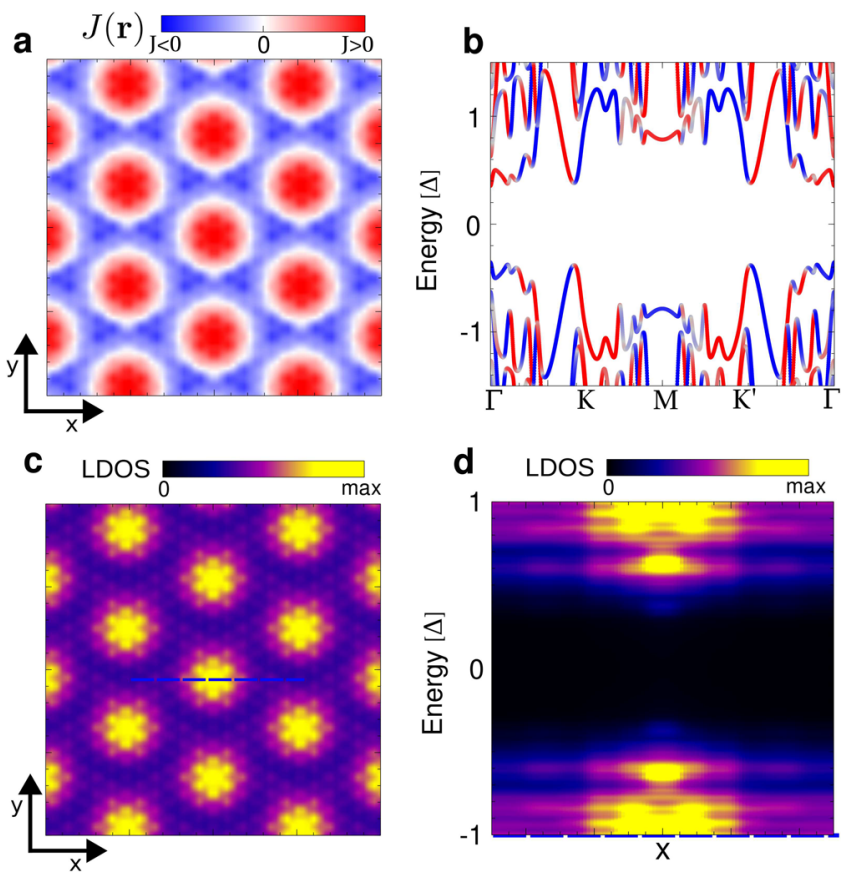

Figure 3. Moiré-enabled topological superconductivity: mechanism in 2D topological superconductor $\mathrm{NbSe}_{2} / \mathrm{CrBr}_{3}$. (a) Moiré profile for the exchange coupling $J(\mathbf{r})$. (b) Resulting band structure in the topological regime with spatially varying exchange as described in the text. (c) Modulations of the YSR band LDOS due to the modulated exchange. (d) Spatial modulation of the bulk YSR bands along the line indicated in $(\mathrm{c})$. additional short-range modulation in both the hopping and local onsite energies. ${ }^{40,41}$ While all these effects can be incorporated into the effective model, we can reproduce the experimental results even using a minimal model that only incorporates spatially varying exchange interactions and onsite energies (Figure 3a). This is supported by the fact that a simple triangular lattice nearest neighbor tight binding model gives a good representation of the Fermi surface of $\mathrm{NbSe}_{2}$ in the presence of Ising and Rashba SOCs and the CDW reconstruction (see Supporting Information section "Realistic tight-binding model for $\mathrm{CrBr}_{3} / \mathrm{NbSe}_{2}$ heterostructure" for details). This results in a topological superconducting band structure (Figure $3 \mathrm{~b}$ ) in a chemical potential range close to charge neutrality, where the system is trivial in the absence of moiré modulations. Associated with these modulations, moirémodulated YSR bands emerge (Figure 3c). The theory predicts (in agreement with our experimental results) that the moire pattern gives rise to a spatial modulation of the intensity (Figure $3 \mathrm{~d}$ ) of the in-gap states but not of their energies.

Above, we have focused on the impact of the moire pattern on the bulk electronic structure, but the moire electronic structure also gets imprinted on the topological edge modes. In particular, the emergence of a YSR moire band structure suggests that topological edge modes may inherit the moire distribution of the bulk YSR states. We now focus on the edge of a $\mathrm{CrBr}_{3}$ island, as shown in Figure 4a. At biases at and above the YSR bands (Figure $4 \mathrm{~b}$ ), no strong modulation at the edge is observed. In stark contrast, when taking energies inside the topological gap, we observe topological edge modes with a strong modulation with the period of the moire pattern (Figure $4 c)$. This is also visible in the single $d I / d V$ spectra extracted at
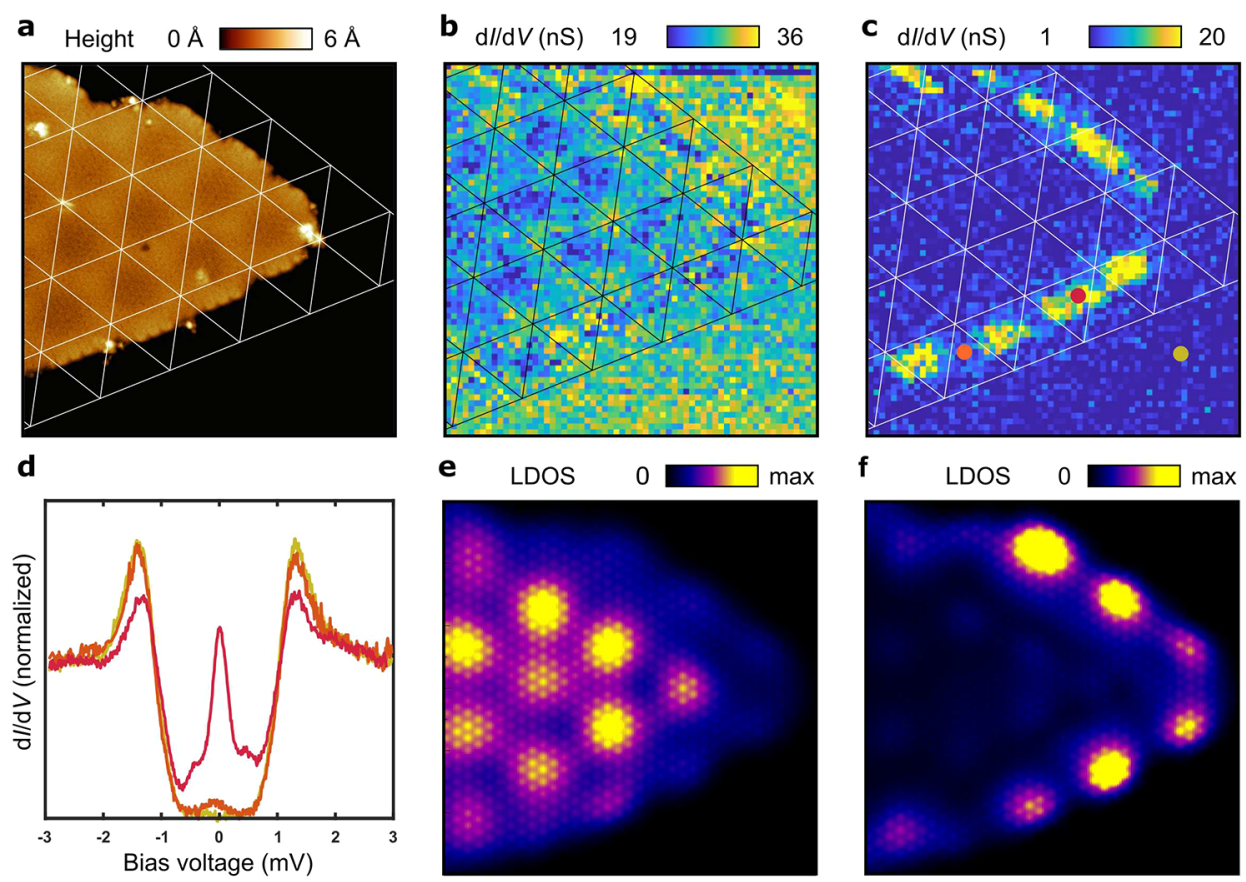

Figure 4. Edge states of the topological superconductor in van der Waals heterostructure with a moire pattern. (a) $33 \times 33 \mathrm{~nm}^{2}$ STM image of $\mathrm{CrBr}_{3}$ island on $\mathrm{NbSe}_{2}$, obtained at $V=1.0 \mathrm{~V}$ and $I=10 \mathrm{pA}$. (b) $\mathrm{d} I / \mathrm{d} V$ map at energy of the YSR bands $V=0.8 \mathrm{mV}$ with a moire pattern appearing in the bulk of $\mathrm{CrBr}_{3}$ island. (c) $\mathrm{d} I / \mathrm{d} V$ map (from spectral grid) at $V=0 \mathrm{mV}$ showing Majorana edge modes. (d) $\mathrm{d} I / \mathrm{d} V$ spectra acquired at the positions indicated in (c) (red, strong edge mode intensity; orange, weak edge mode intensity; yellow, background spectrum on $\mathrm{NbSe}_{2}$. (e, $\mathrm{f}$ ) Theorically computed LDOS in the presence of a moire exchange, at the energies of the YSR states (e) and the Majorana zero modes (f) for an island that closely mimics the shape of the studied experimentally. The grid map measurement conditions are the following: $V=3 \mathrm{mV}, I=140 \mathrm{pA}$, and $T=350 \mathrm{mK}$. 
the points corresponding to the minimum and maximum intensity along the edge (points marked in Figure 4c, spectra shown in Figure 4d). We have analyzed a corresponding finitesize structure with our theoretical model (details in the Supporting Information) as shown in Figure 4e,f. As expected, at energies above the topological gap, modulated YSR states appear (Figure 4e). In strong correspondence with the experimental results, inside the gap, strongly modulated ingap modes dominate the spectra (Figure 4f). This direct relationship between the edge modes and the bulk moire modulation demonstrates a nontrivial role of the moire pattern in creating the topological superconducting state. The moireinduced topological phase transition and the modulation of edge modes are general features of the physical picture and will occur for the one-dimensional and two-dimensional realizations of these systems. This provides an experimentally simple way of verifying the presence and assessing the impact of the moire modulation on the topological superconducting state.

To summarize, we have demonstrated that moiré modulations allow realization of topological superconductivity in parameter regimes otherwise forbidden by the electronic structure. In particular, by accounting for the moire modulation, we have solved three open questions on the emergence of topological superconductivity in $\mathrm{CrBr}_{3} / \mathrm{NbSe}_{2}$. First, the spatial modulation of the edge modes directly corresponds to the moire modulation of the bulk Yu-ShibaRusinov bands. Second, there is no need for the fine-tuning of the chemical potential as the moire modulation results in a topological phase around charge neutrality over a broad range of the values of the chemical potential. And third, the detrimental effect of the Ising spin-orbit interaction is mostly removed by the modification of the band structure due to the charge-density wave modulation of the $\mathrm{NbSe}_{2}$. Concomitant to this moiré-enabled superconducting state, moiré-modulated YSR bands appear, whose topological band structure is ultimately responsible for the topological superconducting state. We have demonstrated this idea in a $\mathrm{CrBr}_{3} / \mathrm{NbSe}_{2}$ twisted heterostructure, showing the emergence of moiré YSR bands and moiré-modulated edge modes, the two paradigmatic experimental signatures of a moiré-enabled topological state. Moiré-enabled topological phase transitions are especially powerful in twisted van der Waals heterostructures, where the twist angle can be used as a knob to push the system to a topological superconducting state. Our results demonstrate the possibility of using twist engineering to design topological quantum materials with a high potential for creating a platform for realizing strongly interacting topological superconductors. This provides a new paradigmatic direction in the field of topological twistronics.

\section{ASSOCIATED CONTENT}

\section{SI Supporting Information}

The Supporting Information is available free of charge at https://pubs.acs.org/doi/10.1021/acs.nanolett.1c03856.

Experimental methods and additional experimental and theoretical results (PDF)

\section{AUTHOR INFORMATION}

\section{Corresponding Authors}

Shawulienu Kezilebieke - Department of Applied Physics, Aalto University, 00076 Aalto, Finland; Department of Physics, Department of Chemistry, and Nanoscience Center,
University of Jyväskylä, FI-40014 Jyväskylä, Finland;

다이.org/0000-0003-4166-5079;

Email: kezilebieke.a.shawulienu@jyu.fi

Jose L. Lado - Department of Applied Physics, Aalto University, 00076 Aalto, Finland; 이이.org/0000-00029916-1589; Email: jose.lado@aalto.fi

\section{Authors}

Viliam Vaňo - Department of Applied Physics, Aalto University, 00076 Aalto, Finland

Md N. Huda - Department of Applied Physics, Aalto University, 00076 Aalto, Finland

Markus Aapro - Department of Applied Physics, Aalto University, 00076 Aalto, Finland

Somesh C. Ganguli - Department of Applied Physics, Aalto University, 00076 Aalto, Finland; 10 orcid.org/0000-00024709-6439

Peter Liljeroth - Department of Applied Physics, Aalto University, 00076 Aalto, Finland; (1) orcid.org/0000-00031253-8097

Complete contact information is available at:

https://pubs.acs.org/10.1021/acs.nanolett.1c03856

\section{Notes}

The authors declare no competing financial interest.

\section{ACKNOWLEDGMENTS}

This research made use of the Aalto Nanomicroscopy Center (Aalto NMC) facilities and was supported by the European Research Council (Grant ERC-2017-AdG No. 788185 "Artificial Designer Materials"), Academy of Finland (Academy Professor Funding Grants 318995 and 320555, Academy Research Fellow Grants 331342, 336243, 338478, and 346654), and the Jane and Aatos Erkko Foundation. We acknowledge the computational resources provided by the Aalto Science-IT project.

\section{REFERENCES}

(1) Beenakker, C. Search for Majorana Fermions in Superconductors. Annu. Rev. Condens. Matter Phys. 2013, 4, 113-136.

(2) Alicea, J. New directions in the pursuit of Majorana fermions in solid state systems. Rep. Prog. Phys. 2012, 75, 076501.

(3) Qi, X.-L.; Zhang, S.-C. Topological insulators and superconductors. Rev. Mod. Phys. 2011, 83, 1057-1110.

(4) Alicea, J.; Oreg, Y.; Refael, G.; von Oppen, F.; Fisher, M. P. A. Non-Abelian statistics and topological quantum information processing in 1D wire networks. Nat. Phys. 2011, 7, 412-417.

(5) Aasen, D.; Hell, M.; Mishmash, R. V.; Higginbotham, A.; Danon, J.; Leijnse, M.; Jespersen, T. S.; Folk, J. A.; Marcus, C. M.; Flensberg, K.; Alicea, J. Milestones Toward Majorana-Based Quantum Computing. Phys. Rev. X 2016, 6, 031016.

(6) Nadj-Perge, S.; Drozdov, I. K.; Li, J.; Chen, H.; Jeon, S.; Seo, J.; MacDonald, A. H.; Bernevig, B. A.; Yazdani, A. Observation of Majorana fermions in ferromagnetic atomic chains on a superconductor. Science 2014, 346, 602-607.

(7) Feldman, B. E.; Randeria, M. T.; Li, J.; Jeon, S.; Xie, Y.; Wang, Z.; Drozdov, I. K.; Bernevig, B. A.; Yazdani, A. High-resolution studies of the Majorana atomic chain platform. Nat. Phys. 2017, 13, 286-291.

(8) Pientka, F.; Glazman, L. I.; von Oppen, F. Topological superconducting phase in helical Shiba chains. Phys. Rev. B 2013, 88,155420 .

(9) Röntynen, J.; Ojanen, T. Topological Superconductivity and High Chern Numbers in 2D Ferromagnetic Shiba Lattices. Phys. Rev. Lett. 2015, 114, 236803. 
(10) Pöyhönen, K.; Ojanen, T. Superlattice platform for chiral superconductivity with tunable and high Chern numbers. Phys. Rev. B 2017, 96, 174521.

(11) Sau, J. D.; Lutchyn, R. M.; Tewari, S.; Das Sarma, S. Generic New Platform for Topological Quantum Computation Using Semiconductor Heterostructures. Phys. Rev. Lett. 2010, 104, 040502.

(12) Oreg, Y.; Refael, G.; von Oppen, F. Helical Liquids and Majorana Bound States in Quantum Wires. Phys. Rev. Lett. 2010, 105, 177002.

(13) Kezilebieke, S.; Huda, M. N.; Vaňo, V.; Aapro, M.; Ganguli, S. C.; Silveira, O. J.; Głodzik, S.; Foster, A. S.; Ojanen, T.; Liljeroth, P. Topological superconductivity in a van der Waals heterostructure. Nature 2020, 588, 424-428.

(14) Mourik, V.; Zuo, K.; Frolov, S. M.; Plissard, S. R.; Bakkers, E. P. A. M.; Kouwenhoven, L. P. Signatures of Majorana Fermions in Hybrid Superconductor-Semiconductor Nanowire Devices. Science 2012, 336, 1003-1007.

(15) Das, A.; Ronen, Y.; Most, Y.; Oreg, Y.; Heiblum, M.; Shtrikman, H. Zero-bias peaks and splitting in an $\mathrm{Al}-\mathrm{InAs}$ nanowire topological superconductor as a signature of Majorana fermions. Nat. Phys. 2012, 8, 887-895.

(16) Deng, M. T.; Vaitiekenas, S.; Hansen, E. B.; Danon, J.; Leijnse, M.; Flensberg, K.; Nygård, J.; Krogstrup, P.; Marcus, C. M. Majorana bound state in a coupled quantum-dot hybrid-nanowire system. Science 2016, 354, 1557-1562.

(17) Ugeda, M. M.; Bradley, A. J.; Zhang, Y.; Onishi, S.; Chen, Y.; Ruan, W.; Ojeda-Aristizabal, C.; Ryu, H.; Edmonds, M. T.; Tsai, H.Z.; Riss, A.; Mo, S.-K.; Lee, D.; Zettl, A.; Hussain, Z.; Shen, Z.-X.; Crommie, M. F. Characterization of collective ground states in singlelayer $\mathrm{NbSe}_{2}$. Nat. Phys. 2016, 12, 92-97.

(18) Rokhinson, L. P.; Liu, X.; Furdyna, J. K. The fractional a.c. Josephson effect in a semiconductor-superconductor nanowire as a signature of Majorana particles. Nat. Phys. 2012, 8, 795-799.

(19) Cai, X.; Lang, L.-J.; Chen, S.; Wang, Y. Topological Superconductor to Anderson Localization Transition in OneDimensional Incommensurate Lattices. Phys. Rev. Lett. 2013, 110, 176403.

(20) DeGottardi, W.; Sen, D.; Vishveshwara, S. Majorana Fermions in Superconducting 1D Systems Having Periodic, Quasiperiodic, and Disordered Potentials. Phys. Rev. Lett. 2013, 110, 146404.

(21) Malard, M.; Japaridze, G. I.; Johannesson, H. Synthesizing Majorana zero-energy modes in a periodically gated quantum wire. Phys. Rev. B 2016, 94, 115128.

(22) Tezuka, M.; Kawakami, N. Reentrant topological transitions in a quantum wire/superconductor system with quasiperiodic lattice modulation. Phys. Rev. B 2012, 85, 140508.

(23) Levine, Y.; Haim, A.; Oreg, Y. Realizing topological superconductivity with superlattices. Phys. Rev. B 2017, 96, 165147.

(24) Bøttcher, C. G. L.; Nichele, F.; Kjaergaard, M.; Suominen, H. J.; Shabani, J.; Palmstrøm, C. J.; Marcus, C. M. Superconducting, insulating and anomalous metallic regimes in a gated two-dimensional semiconductor-superconductor array. Nat. Phys. 2018, 14, 11381144.

(25) Laeven, T.; Nijholt, B.; Wimmer, M.; Akhmerov, A. R. Enhanced Proximity Effect in Zigzag-Shaped Majorana Josephson Junctions. Phys. Rev. Lett. 2020, 125, 086802.

(26) Harper, P. G. Single Band Motion of Conduction Electrons in a Uniform Magnetic Field. Proc. Phys. Soc. A 1955, 68, 874-878.

(27) Lutchyn, R. M.; Sau, J. D.; Das Sarma, S. Majorana Fermions and a Topological Phase Transition in Semiconductor-Superconductor Heterostructures. Phys. Rev. Lett. 2010, 105, 077001.

(28) Thouless, D. J.; Kohmoto, M.; Nightingale, M. P.; den Nijs, M. Quantized Hall Conductance in a Two-Dimensional Periodic Potential. Phys. Rev. Lett. 1982, 49, 405-408.

(29) Bistritzer, R.; MacDonald, A. H. Moire bands in twisted double-layer graphene. Proc. Nat. Acad. Sci. 2011, 108, 12233-12237.

(30) Suárez Morell, E.; Correa, J. D.; Vargas, P.; Pacheco, M.; Barticevic, Z. Flat bands in slightly twisted bilayer graphene: Tightbinding calculations. Phys. Rev. B 2010, 82, 121407.
(31) Algra, R. E.; Verheijen, M. A.; Borgström, M. T.; Feiner, L.-F.; Immink, G.; van Enckevort, W. J. P.; Vlieg, E.; Bakkers, E. P. A. M. Twinning superlattices in indium phosphide nanowires. Nature 2008, 456, 369-372.

(32) Zhang, G.; Takiguchi, M.; Tateno, K.; Tawara, T.; Notomi, M.; Gotoh, H. Telecom-band lasing in single InP/InAs heterostructure nanowires at room temperature. Sci. Adv. 2019, 5, No. eaat8896.

(33) Kezilebieke, S.; Silveira, O. J.; Huda, M. N.; Vaňo, V.; Aapro, M.; Ganguli, S. C.; Lahtinen, J.; Mansell, R.; van Dijken, S.; Foster, A. S.; Liljeroth, P. Electronic and Magnetic Characterization of Epitaxial $\mathrm{CrBr}_{3}$ Monolayers on a Superconducting Substrate. Adv. Mater. 2021, 33, 2006850.

(34) Chen, W.; Sun, Z.; Wang, Z.; Gu, L.; Xu, X.; Wu, S.; Gao, C. Direct observation of van der Waals stacking-dependent interlayer magnetism. Science 2019, 366, 983-987.

(35) Sivadas, N.; Okamoto, S.; Xu, X.; Fennie, C. J.; Xiao, D. Stacking-Dependent Magnetism in Bilayer $\mathrm{CrI}_{3}$. Nano Lett. 2018, 18, 7658-7664.

(36) Song, T.; Fei, Z.; Yankowitz, M.; Lin, Z.; Jiang, Q.; Hwangbo, K.; Zhang, Q.; Sun, B.; Taniguchi, T.; Watanabe, K.; McGuire, M. A.; Graf, D.; Cao, T.; Chu, J.-H.; Cobden, D. H.; Dean, C. R.; Xiao, D.; $\mathrm{Xu}, \mathrm{X}$. Switching $2 \mathrm{D}$ magnetic states via pressure tuning of layer stacking. Nat. Mater. 2019, 18, 1298-1302.

(37) Wickramaratne, D.; Khmelevskyi, S.; Agterberg, D. F.; Mazin, I. I. Ising Superconductivity and Magnetism in $\mathrm{NbSe}_{2}$. Phys. Rev. $X$ 2020, 10, 041003.

(38) Naik, M. H.; Jain, M. Ultraflatbands and Shear Solitons in Moiré Patterns of Twisted Bilayer Transition Metal Dichalcogenides. Phys. Rev. Lett. 2018, 121, 266401.

(39) Enaldiev, V. V.; Zólyomi, V.; Yelgel, C.; Magorrian, S. J.; Fal'ko, V. I. Stacking Domains and Dislocation Networks in Marginally Twisted Bilayers of Transition Metal Dichalcogenides. Phys. Rev. Lett. 2020, 124, 206101.

(40) Zheng, F.; Zhou, Z.; Liu, X.; Feng, J. First-principles study of charge and magnetic ordering in monolayer $\mathrm{NbSe}_{2}$. Phys. Rev. B 2018, 97, 081101.

(41) Guster, B.; Rubio-Verdú, C.; Robles, R.; Zaldívar, J.; Dreher, P.; Pruneda, M.; Silva-Guillén, J. Á.; Choi, D.-J.; Pascual, J. I.; Ugeda, M. M.; Ordejón, P.; Canadell, E. Coexistence of Elastic Modulations in the Charge Density Wave State of $2 \mathrm{H}-\mathrm{NbSe}_{2}$. Nano Lett. 2019, 19, 3027-3032. 